\title{
Klassifikationsschema für Usability-Evaluationsmethoden
}

\author{
Christoph Ohl ${ }^{1}$, Gabriele Schade ${ }^{2}$ \\ Institut für Informatik, Friedrich-Schiller-Universität Jena ${ }^{1}$ \\ Fachrichtung Angewandte Informatik, Fachhochschule Erfurt ${ }^{2}$
}

\section{Zusammenfassung}

Nicht immer ist innerhalb von Software-Projekten das notwendige Usability-Wissen zur zielgerichteten und effizienten Auswahl geeigneter Methoden vorhanden. Zur Unterstützung eines Auswahlprozesses, welche Methode ist wann die am besten geeignete, ist es von Vorteil, wenn das Wissen über die Methoden in strukturierter Form vorliegt und angemessene Kriterien zur Unterscheidung gefunden werden können. Bisherige diesbezügliche Lösungen sind hier nur bedingt zur Unterstützung des Auswahl-prozesses geeignet. In diesem Beitrag wird ein Klassifikationsschema vorgeschlagen, dass der Ein- und Zuordnung von Usability-Evaluationsmethoden dienen soll und einen Ausgangspunkt zur besseren Auswahl geeigneter Verfahren im Usability-Engineering darstellen kann.

\section{Einleitung}

Zur Bewertung von Mensch-Maschine-Schnittstellen existieren mittlerweile unzählige Usability-Evaluationsmethoden, die sich jeweils für bestimmte Einsatzzwecke eignen und im Vergleich zueinander ganz verschiedene Charakteristika aufweisen. Durch die große Anzahl und die zum Teil doch eher formlosen Beschreibungen dieser Methoden seitens der Autoren wird es immer schwieriger, deren Unterschiede und Gemeinsamkeiten zu überblicken. Eine genauere Betrachtung der Methodenvielfalt lässt den Schluss zu, dass die Auswahl von geeigneten Usability-Methoden für einen konkreten Anwendungsfall keine triviale Aufgabe ist und dazu eine entsprechende Expertise vorliegen muss. Häufig sind in den (vielfach auch kleinen und mittelständischen) Unternehmen aber keine Usability-Experten vorhanden. So schätzen die mittelständischen Unternehmen in Deutschland im Jahre $2011 \mathrm{ihr}$ Wissen zu Usability-Methoden als eher gering ein (Woywode et al. 2012). Da für eine planvolle Auswahl eine gewisse Methodenkompetenz und somit grundlegendes Überblickswissen über die bestehenden Methoden notwendig ist, kann davon ausgegangen werden, dass die Auswahl der Methoden im Usability-Engineering bei mittelständischen Produzenten in Deutschland 
aufgrund fehlender Methodenkenntnis meist ohne zugrundeliegende Systematik erfolgt. Während die Methodenvielfalt wächst, besteht die Aufgabe seitens der Experten nun darin, eine gemeinsame Wissensbasis und somit ein größeres Verständnis im Hinblick auf die einzelnen Verfahren zu schaffen. Die Grundlage einer solchen Wissenssammlung setzt unter anderem eine genaue Definition von Kriterien zur Unterscheidung sowie in diesem Zusammenhang ein gut strukturiertes Klassifikationsschema zur Einordnung der Methoden voraus. In der Literatur lassen sich bereits von Experten veröffentlichte Klassifikationsschemen zur Ordnung von Usability-Methoden finden (Siehe Kapitel 2). Diese stellen gute Lösungen dar, um die Methoden logisch zu gruppieren und um ein entsprechendes Überblickswissen zu vermitteln. Es werden jedoch nur eine relativ geringe Anzahl von Merkmalen zur Klassifikation genutzt. Die Vergleichbarkeit der Methoden unter Zuhilfenahme der Klassifikationsschemen ist dabei auf wenige Unterscheidungsmerkmale limitiert.

Der vorliegende Beitrag bietet eine Klassifikationslösung an, die sich zum Aufbau einer Wissenssammlung zu den Methoden der Usability-Evaluation eignet und Potenzial besitzt, unterstützend bei der Auswahl geeigneter Evaluationsverfahren im Usability-Engineering zu sein. Letztendlich kann auf dieser Grundlage ein Instrumentarium entstehen (z. B. digitalen Assistenten), das sowohl für Neueinsteiger im Themenfeld Usability oder für UsabilityExperten eine Hilfe darstellt. Je nach Adressat des Instrumentariums kann die letztendliche Ausgestaltung stark variieren (z. B. Nutzung von Begrifflichkeiten und Abfolge der Arbeitsschritte).

\section{Verwandte Arbeiten}

Es existiert eine Reihe von Veröffentlichungen zu Klassifikationsschemen zur Ordnung von Usability-Evaluationsmethoden. Für eine detaillierte Beschreibung der einzelnen Klassifikationen, sei an dieser Stelle auf die zugehörigen Literaturangaben verwiesen (Whitefield et al. 1991; Ivory \& Hearst 2001; Christos et al. 2007; Zhang 2007, Law et al. 2009, S.7 ff.; Seffah \& Metzker 2009, S.17 f.; Krannich 2010; Sarodnick \& Brau 2011). Eine aufbereitete Gegenüberstellung dieser Klassifikationen in tabellarischer Form ist in Ohl (2014) zu finden. Bei näherer Betrachtung wird deutlich, dass die Mehrzahl der existierenden Klassifikationssysteme bezüglich der Basisklassen einen mehr oder weniger ähnlichen Aufbau aufweist. Je nach Autoren variieren die Bezeichnungen. Die Klassen werden entweder aggregiert oder weiter untergliedert. Dennoch lassen sich bei der Gegenüberstellung deutlich mehr Gemeinsamkeiten als Unterschiede erkennen. In den meisten Fällen wird danach klassifiziert, in welcher Art und Weise die Nutzer und das zu untersuchende System am Evaluationsprozess beteiligt sind. Sehr deutlich wird diese Differenzierung beispielsweise in der Kreuzklassifikation von Whitefield und Kollegen (siehe Abbildung 1). Neben den inhaltlichen Aspekten sind Unterschiede und Gemeinsamkeiten hinsichtlich der grundsätzlichen Struktur erkennbar. Die Mehrheit der gefundenen Lösungen zur Klassifikation verwendet eine präkombinierte und eindimensionale hierarchische Struktur. Diese eignen sich sehr gut für eine grobe und einfach zu verstehende Unterteilung bzw. Einsortierung der Methoden. Daneben existieren aber auch Lösungen, die eine mehrdimensionale Struktur aufweisen und somit umfangreichere Gliederungen und Einordnungen erlauben (Whitefield et al. 1991; Ivory \& Hearst 
2001; Law et al. 2009; Sarodnick \& Brau 2011). Eine Klassifikation, die sich dabei von den restlichen abhebt, bieten Ivory und Hearst (2001). Dabei handelt es sich um eine Postkombinierte Klassifikation bzw. Facettenklassifikation, bei der neben der allgemeinen Methodenklasse („Method Class“) und dem konkreten Methodentypen („Method Type“), ebenfalls nach dem Grad der technischen Unterstützung („Automation Type“) sowie nach dem Aufwand zur Durchführung der Methode („Effort Level“) klassifiziert wird. Die Vorteile beim Einsatz von Facetten werden hier bereits sehr deutlich, was auch zur Inspiration des eigenen Lösungsansatzes diente, der in den nachfolgenden Kapiteln erläutert wird.

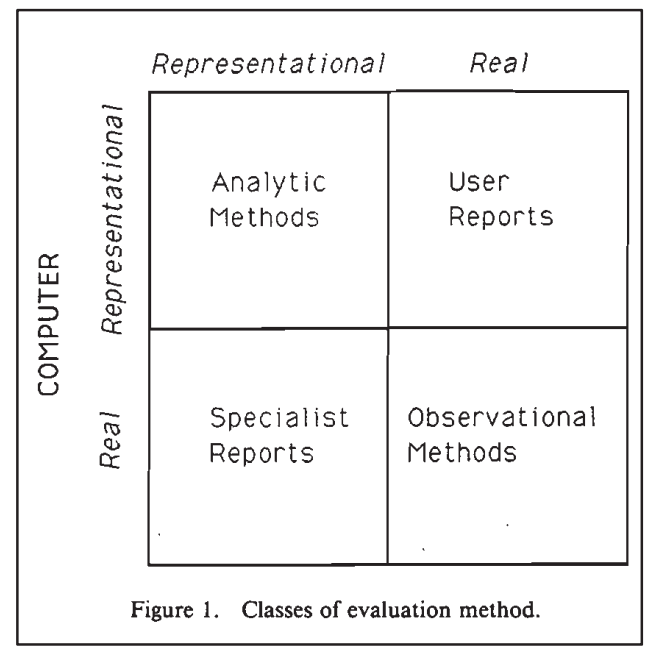

Abbildung 1: Kreuzklassifikation von Usability-Evaluationsmethoden nach Whitefield et al. (1991)

\section{Anforderungen}

Im Folgenden werden die von den Autorinnen und Autoren dieses Beitrags benannten Anforderungen (Siehe Kapitel 1) an ein Klassifikationsschema formuliert, welches es erlaubt, gut zwischen einzelnen Usability-Evaluationsmethoden zu differenzieren und zugleich eine Entscheidungshilfe zur Auswahl geeigneter Evaluationsverfahren darstellt.

- Abgrenzung: Die Abgrenzung zu anderen Methoden im Themenbereich Usability, also solche die nicht im direkten Zusammenhang mit der Evaluation eines Untersuchungsgegenstandes stehen, muss erkenntlich sein.

- Flexibilität: Das Klassifikationsschema soll neben der rationellen Handhabung von Wissen über die Methoden auch der Wissenserkenntnis dienen. Dafür sollten auch komplexe Sachverhalte dargestellt werden können und verschiedene Sichtweisen auf die Methoden realisierbar sein. Eine mögliche Fragestellung mit einem vergleichsweise geringerem Komplexitätsgrad, die in diesem Kontext beantwortet werden könnte, lautet beispielsweise wie folgt: Gibt es UsabilityEvaluationsmethoden, die mir quantifizierbare Ergebnisse über wenige, ausgesuchte 
Untersuchungskriterien liefern, wenn ich eine abschließende Bewertung eines Designergebnisses (Mockup) anstrebe, dass in den frühen Phasen des Projektes entstand?

- Pflege und Erweiterbarkeit: Das Klassifikationsschema soll leicht erweitert oder geändert werden können.

- Einfache Anwendbarkeit: Das Klassifikationsschema soll einfach anzuwenden sein und gute Selektionsmöglichkeiten über alle erfassten Methoden bieten.

\section{Klassifikationsschema}

\subsection{Vorgehensweise}

Zur Erfüllung der zuvor definierten Anforderungen wurde die Entwicklung einer postkombinierten Klassifikation angestrebt. Dabei zeigte sich die Facettenklassifikation als gut geeignet, weil sie es erlaubt, die Methoden der Usability-Evaluation aus verschiedenen Blickwinkeln zu betrachten und so große Mengen von Elementen sinnvoll zu differenzieren. Der eigentlichen Klassifizierung ging eine Analyse der Usability-Evaluationsmethoden, also der zu klassifizierenden Elemente, voraus. Hierbei ist als Bezugsquelle die in Kapitel 2 vorgestellten Klassifikationssysteme sowie weitere Fachliteratur $\mathrm{zu}$ diesem Themengebiet $\mathrm{zu}$ nennen (allen voran Sarodnick \& Brau 2011; Freymann 2008; Bortz \& Döring 2006). Anschließend fand eine Unterteilung der Methoden entsprechend ihrer Merkmale in Einfachklassen statt. Daraufhin erfolgte die Kombination und Zusammenfassung der klassifizierten Merkmale zu mehreren gleichrangigen, voneinander unabhängigen, Kategorien, den Facetten.

\subsection{Facettenanalyse}

Es konnten mehrere Merkmale bestimmt werden, die sich schließlich zur Klassifizierung der Methoden eignen. Daraus ließen sich einzelne Klassen ableiten, die wiederum in Facetten eingruppiert wurden. Grundsätzlich lassen sich hierbei zwei Arten von Facetten unterscheiden: Facetten, die eine dimensionale Klassifizierung erlauben und dadurch auf Dimensionen wie Ort und Zeit abbildbar sind und Facetten, die eine kategoriale Klassifizierung unterliegen und zur Abgrenzung voneinander hinsichtlich bestimmter Merkmale dienen. Facetten mit einer dimensionalen Klassifizierung sind für direkte Auswahlprozesse als zweitrangig einzustufen. Letztere bieten aber bei der dokumentarischen Betrachtung der Methoden im historischen Kontext einen hohen Mehrwert und werden daher ergänzend aufgeführt. Die zusammengetragenen Facetten werden nun kurz vorgestellt. 


\section{Dimensionale Klassifizierung}

A. Erscheinungsjahr / Release Date: Jede Methode wurde zu einem bestimmten Zeitpunkt erstmals publiziert und damit öffentlich bekannt gemacht. Das Datum der Veröffentlichung erlaubt die Klassifizierung hinsichtlich der zeitlichen Einordnung der Methoden.

B. Entwicklungsstandort / Development site: Jede Methode kann einem Entwicklungsstandort zugeordnet werden. Dieser Ort erlaubt eine Klassifizierung hinsichtlich der geographischen Lage der Methoden.

C. Erfinder oder Herausgeber / Developer or publisher: Jede Methode wurde von einer oder mehreren Personen erfunden bzw. erstmals publiziert. Dadurch ist eine Klassifizierung hinsichtlich der Erfinder der Methoden möglich.

\section{Kategoriale Klassifizierung}

D. Untersuchungszweck / Purpose of investigation: Grundsätzlich lassen sich zwei Evaluationsformen gegenüberstellen: die formative und die summative Evaluation (Sarodnick \& Brau 2011). Methoden, die bereits während eines Designprozesses angewandt werden können und auf ein direktes Feedback zur Optimierung des Untersuchungsgegenstandes abzielen, zählen zur formativen Evaluation. Meist werden diese Evaluationsverfahren iterativ eingesetzt und dienen zur Qualitätssicherung. Im Gegensatz dazu stehen Methoden, die der summativen Evaluation zugeordnet werden und erst nach dem Abschluss eines Designprozesses anwendbar sind. Dadurch erlauben sie eine zusammenfassende oder vergleichende Bewertung des zu untersuchenden Systems. Diese Evaluationsverfahren dienen somit vorrangig der Qualitätskontrolle. Es existieren etliche Methoden, die sich sowohl zur formativen als auch zur summativen Evaluation anbieten.

E. Untersuchungskriterien / Test criteria: Die Usability als solche lässt sich in einzelne Prüfkriterien zerlegen (DIN ISO 9241-11 1998). Viele Methoden verfolgen einen holistischen Ansatz, bei denen allgemeine Aussagen über die Usability eines Systems angestrebt werden. Andere Methoden beziehen sich bei der Prüfung wiederum auf bestimmte Kriterien der Usability, weshalb in solchen Fällen von einer partiellen Prüfung der Usability ausgegangen werden kann. Eine weitere Unterteilung der partiellen Prüfung in einzelne Usability-Kriterien ist möglich und erscheint sinnvoll. Da der Qualitätsbegriff Usability nach Meinung der Autorinnen und Autoren dieses Beitrags aber noch nicht erschöpfend definiert wurde und es noch Diskussionsbedarf seitens der Experten bedarf, können an dieser Stelle keine festen Kriterien mit eingebracht werden.

F. Untersuchungsgegenstand / Object of investigation: Die Anwendbarkeit einer Methode ist von verschiedenen Vorbedingungen abhängig. Entscheidend ist zum Beispiel die Form, in der ein Untersuchungsgegenstand vorliegt (Freymann 2008). Während bei einigen Methoden konzeptionelle Beschreibungen oder Papierskizzen ausreichen, sind für andere wiederum prototypische Umsetzungen oder sogar voll funktionsfähige Systeme notwendig. 
G. Präsenz des Untersuchungsgegenstandes / Presence of the object of investigation: Generell kann danach unterschieden werden, in welcher Art der Untersuchungsgegenstand während des Evaluationsprozesses beteiligt ist. Whitefield und Kollegen unterscheiden hierbei zwischen „real“ und „,repräsentativ“ (Whitefield et. al. 1991). Dabei ist die Eigenschaft ,real“" so zu verstehen, dass auch Prototypen und Simulationen eines Systems in gewisser Weise als reales Untersuchungsobjekt zählen. Im Gegensatz dazu können für eine Evaluation auch Modelle und Spezifikationen genutzt werden, die eine mentale Repräsentation des Untersuchungsgegenstandes darstellen und demnach der Untersuchungsgegenstand nur ,repräsentativ“ zur Verfügung steht.

H. Gutachter / Evaluator: Eine Einteilung der Methoden ist in Abhängigkeit davon möglich, wer an der Untersuchung beteiligt ist bzw. wer als Gutachter fungiert. Bei den empirischen bzw. benutzerbasierten Verfahren werden die Informationen über Befragung und Beobachtung der tatsächlichen oder potentiellen Nutzer gewonnen. Bei den analytischen bzw. expertenbasierten Verfahren werden hingegen die Beurteilung von Usability-Experten vorgenommen, die versuchen, sich in die Situation der echten Nutzer zu versetzen (Sarodnick \& Brau 2011: 119f.).

I. Analyseform / Forms of analysis: Die Evaluationsverfahren können danach betrachtet werden, auf welche Art und Weise die Untersuchungsergebnisse ermittelt werden. Dabei wird zwischen qualitativen und quantitativen Methoden unterschieden (Bortz \& Döring 2006: 137ff.). Qualitative Methoden beinhalten Ergebnisbeschreibungen in verbaler Form, die typischerweise detailliert, ganzheitlich und umfassend sind. Quantitative Methoden liefern hingegen Ergebnisse über wenige, ausgesuchte Untersuchungskriterien, welche systematisch operationalisiert bzw. quantifiziert werden. Während quantitative Untersuchungen objektive Erkenntnisse auf breiter Basis liefern, erzielt das qualitative Vorgehen tiefere Einsichten zu einem Untersuchungsaspekt, wobei in einigen Fällen ein hohes Maß an Interpretation nötig ist. Bei vielen Methoden werden sowohl qualitative als auch quantitative Daten erhoben.

J. Anwendungsdomäne / Application domain: Die meisten Evaluationsmethoden und techniken zur Überprüfung der Usability sind so allgemein gehalten, dass sie für verschiedene Anwendungsdomänen gleichermaßen und somit universell einsetzbar sind. Die Evaluationsmethoden müssen ggf. noch an dem jeweiligen Kontext der Untersuchung angepasst werden, bleiben aber vom prinzipiellen Aufbau unverändert. Im Gegensatz dazu gibt es Methoden, die speziell für eine bestimmte Domäne, wie zum Beispiel einer Web-Anwendung oder einem mobilen System, entwickelt worden sind und sich nicht einfach auf andere Anwendungsfelder übertragen lassen. 


\begin{tabular}{|c|c|c|c|c|c|c|}
\hline Facet & Characteristics & $\mathbf{M A}^{\mathbf{1}}$ & $\begin{array}{l}\text { Example 1: } \\
\text { Cognitive } \\
\text { Walkthrough }\end{array}$ & $\begin{array}{l}\text { Example 2: } \\
\text { Thinking } \\
\text { Aloud Test }\end{array}$ & $\begin{array}{l}\text { Example 3: } \\
\text { Heuristic } \\
\text { Evaluation }\end{array}$ & $\begin{array}{l}\text { Example 4: } \\
\text { Eye-Tracking } \\
\text { Analysis }\end{array}$ \\
\hline \multicolumn{7}{|c|}{ I Dimensional classification (no guarantee of completeness or accuracy) } \\
\hline $\begin{array}{l}\text { A. Release } \\
\text { Date }\end{array}$ & - & No & $\begin{array}{l}\text { January 5, } \\
1992\end{array}$ & $\begin{array}{l}\text { February 17, } \\
1982\end{array}$ & April 4, 1990 & $\begin{array}{l}\text { February } 2, \\
1950\end{array}$ \\
\hline $\begin{array}{l}\text { B. Develop- } \\
\text { ment site }\end{array}$ & - & No & $\begin{array}{l}\text { United States, } \\
\text { Colorado }\end{array}$ & $\begin{array}{l}\text { United States, } \\
\text { New York }\end{array}$ & $\begin{array}{l}\text { Denmark, } \\
\text { Hovedstaden }\end{array}$ & $\begin{array}{l}\text { United States, } \\
\text { Ohio }\end{array}$ \\
\hline $\begin{array}{l}\text { C. Developer } \\
\text { or publisher }\end{array}$ & - & No & $\begin{array}{l}\text { Peter G. } \\
\text { Polson, } \\
\text { Clayton } \\
\text { Lewis, } \\
\text { John Rieman } \\
\text { and Cathleen } \\
\text { Wharton }\end{array}$ & $\begin{array}{l}\text { Clayton } \mathrm{H} . \\
\text { Lewis }\end{array}$ & $\begin{array}{l}\text { Jakob Nielsen } \\
\text { and Rolf } \\
\text { Molich }\end{array}$ & $\begin{array}{l}\text { Paul M. Fitts, , } \\
\text { Richard E. } \\
\text { Jones and John } \\
\text { L. Milton }\end{array}$ \\
\hline \multicolumn{7}{|c|}{ II Categorical classification } \\
\hline $\begin{array}{l}\text { D. Purpose of } \\
\text { investigation }\end{array}$ & $\begin{array}{l}\text { D.1. Summative } \\
\text { evaluation } \\
\text { D.2. Formative } \\
\text { evaluation }\end{array}$ & Yes & $\begin{array}{l}\text { Formative } \\
\text { evaluation }\end{array}$ & $\begin{array}{l}\text { Summative } \\
\text { evaluation, } \\
\text { Formative } \\
\text { evaluation }\end{array}$ & $\begin{array}{l}\text { Summative } \\
\text { evaluation, } \\
\text { Formative } \\
\text { evaluation }\end{array}$ & $\begin{array}{l}\text { Formative } \\
\text { evaluation }\end{array}$ \\
\hline $\begin{array}{l}\text { E. Test } \\
\text { criteria }\end{array}$ & $\begin{array}{l}\text { E.1. Holistic } \\
\text { E.2. Partial }\end{array}$ & No & Partial & Holistic & Holistic & Partial \\
\hline $\begin{array}{l}\text { F. Object of } \\
\text { investigation }\end{array}$ & $\begin{array}{l}\text { F.1. Concept } \\
\text { F.2. Prototyp } \\
\text { F.3. System }\end{array}$ & Yes & $\begin{array}{l}\text { Prototyp, } \\
\text { System }\end{array}$ & $\begin{array}{l}\text { Prototyp, } \\
\text { System }\end{array}$ & $\begin{array}{l}\text { Prototyp, } \\
\text { System }\end{array}$ & $\begin{array}{l}\text { Prototyp } \\
\text { System }\end{array}$ \\
\hline $\begin{array}{l}\text { G. Presence } \\
\text { of the object } \\
\text { of investi- } \\
\text { gation }\end{array}$ & $\begin{array}{l}\text { G.1. Real } \\
\text { G.2. Represen- } \\
\text { tational }\end{array}$ & No & Real & Real & Real & Real \\
\hline H. Evaluator & $\begin{array}{l}\text { H.1. User } \\
\text { H.2. Expert }\end{array}$ & Yes & Expert & User & Expert & User \\
\hline $\begin{array}{l}\text { I. Forms of } \\
\text { analysis }\end{array}$ & $\begin{array}{l}\text { I.1. Quantitative } \\
\text { analysis } \\
\text { I.2. Qualitative } \\
\text { analysis }\end{array}$ & Yes & $\begin{array}{l}\text { Qualitative } \\
\text { analysis }\end{array}$ & $\begin{array}{l}\text { Qualitative } \\
\text { analysis }\end{array}$ & $\begin{array}{l}\text { Qualitative } \\
\text { analysis }\end{array}$ & $\begin{array}{l}\text { Quantitative } \\
\text { analysis }\end{array}$ \\
\hline $\begin{array}{l}\text { J. Application } \\
\text { domain }\end{array}$ & $\begin{array}{l}\text { J.1. Universal } \\
\text { J.2. Special }\end{array}$ & No & Universal & Universal & Universal & Special \\
\hline
\end{tabular}

Tabelle 1: Facettenklassifikation für Usability-Evaluationsmethoden (Beispiel 1 bis 4)

$1 \quad$ Multiple assignment 


\begin{tabular}{|c|c|c|c|c|c|c|}
\hline Facet & Characteristics & MA & $\begin{array}{l}\text { Example 5: } \\
\text { Card Sorting }\end{array}$ & $\begin{array}{l}\text { Example 6: } \\
\text { GOMS }\end{array}$ & $\begin{array}{l}\text { Example 7: } \\
\text { Diary Study }\end{array}$ & $\begin{array}{l}\text { Example 8: } \\
\text { Focus Group }\end{array}$ \\
\hline \multicolumn{7}{|c|}{ I Dimensional classification (no guarantee of completeness or accuracy) } \\
\hline $\begin{array}{l}\text { A. Release } \\
\text { Date }\end{array}$ & - & No & June 22, 1949 & June 27,1983 & April 14, 1993 & May 5, 1946 \\
\hline $\begin{array}{l}\text { B. Develop- } \\
\text { ment site }\end{array}$ & - & No & $\begin{array}{l}\text { United States, } \\
\text { New York }\end{array}$ & $\begin{array}{l}\text { United States, } \\
\text { New York }\end{array}$ & $\begin{array}{l}\text { United States, } \\
\text { Colorado }\end{array}$ & $\begin{array}{l}\text { United States, } \\
\text { New York }\end{array}$ \\
\hline $\begin{array}{l}\text { C. Developer } \\
\text { or publisher }\end{array}$ & - & No & $\begin{array}{l}\text { Cornelius } \\
\text { Dubois }\end{array}$ & $\begin{array}{l}\text { Stuart K. Card, } \\
\text { Thomas P. } \\
\text { Moran and } \\
\text { Allen Newell }\end{array}$ & John Riemann & $\begin{array}{l}\text { Robert K. } \\
\text { Merton and } \\
\text { Patricia L. } \\
\text { Kendall }\end{array}$ \\
\hline \multicolumn{7}{|c|}{ II Categorical classification } \\
\hline $\begin{array}{l}\text { D. Purpose of } \\
\text { investigation }\end{array}$ & $\begin{array}{l}\text { D.1. Summative } \\
\text { evaluation } \\
\text { D.2. Formative } \\
\text { evaluation }\end{array}$ & Yes & $\begin{array}{l}\text { Formative } \\
\text { evaluation }\end{array}$ & $\begin{array}{l}\text { Formative } \\
\text { evaluation }\end{array}$ & $\begin{array}{l}\text { Summative } \\
\text { evaluation }\end{array}$ & $\begin{array}{l}\text { Formative } \\
\text { evaluation }\end{array}$ \\
\hline $\begin{array}{l}\text { E. Test } \\
\text { criteria }\end{array}$ & $\begin{array}{l}\text { E.1. Holistic } \\
\text { E.2. Partial }\end{array}$ & No & Partial & Partial & Holistic & Holistic \\
\hline $\begin{array}{l}\text { F. Object of } \\
\text { investigation }\end{array}$ & $\begin{array}{l}\text { F.1. Concept } \\
\text { F.2. Prototyp } \\
\text { F.3. System }\end{array}$ & Yes & $\begin{array}{l}\text { Concept } \\
\text { Prototyp } \\
\text { System }\end{array}$ & System & System & $\begin{array}{l}\text { Prototyp } \\
\text { System }\end{array}$ \\
\hline $\begin{array}{l}\text { G. Presence } \\
\text { of the object } \\
\text { of investi- } \\
\text { gation }\end{array}$ & $\begin{array}{l}\text { G.1. Real } \\
\text { G.2. Represen- } \\
\text { tational }\end{array}$ & No & $\begin{array}{l}\text { Represen- } \\
\text { tational }\end{array}$ & $\begin{array}{l}\text { Represen- } \\
\text { tational }\end{array}$ & $\begin{array}{l}\text { Represen- } \\
\text { tational }\end{array}$ & $\begin{array}{l}\text { Represen- } \\
\text { tational }\end{array}$ \\
\hline H. Evaluator & $\begin{array}{l}\text { H.1. User } \\
\text { H.2. Expert }\end{array}$ & Yes & User & Expert & User & $\begin{array}{l}\text { User } \\
\text { Expert }\end{array}$ \\
\hline $\begin{array}{l}\text { I. Forms of } \\
\text { analysis }\end{array}$ & $\begin{array}{l}\text { I.1. Quantitative } \\
\text { analysis } \\
\text { I.2. Qualitative } \\
\text { analysis }\end{array}$ & Yes & $\begin{array}{l}\text { Quantitative } \\
\text { analysis } \\
\text { Qualitative } \\
\text { analysis }\end{array}$ & $\begin{array}{l}\text { Quantitative } \\
\text { analysis }\end{array}$ & $\begin{array}{l}\text { Quantitative } \\
\text { analysis } \\
\text { Qualitative } \\
\text { analysis }\end{array}$ & $\begin{array}{l}\text { Qualitative } \\
\text { analysis }\end{array}$ \\
\hline $\begin{array}{l}\text { J. Application } \\
\text { domain }\end{array}$ & $\begin{array}{l}\text { J.1. Universal } \\
\text { J.2. Special }\end{array}$ & No & Universal & Universal & Universal & Universal \\
\hline
\end{tabular}

Tabelle 2: Facettenklassifikation für Usability-Evaluationsmethoden (Beispiel 5 bis 8)

\subsection{Ergebnis}

Aus den bisherigen Überlegungen konnte ein Klassifikationsschema abgeleitet werden, deren zusammengetragenen Facetten sowie deren Ausprägungen, die möglichen Einfachklassen, in Tabelle 1 zu sehen sind. In einigen Fällen können die Methoden zu mehreren Ausprägungen einer Facette zugeordnet werden. Zur Hervorhebung solcher Fälle existiert in Tabelle 1 eine zusätzliche Spalte, die Auskunft über eine mögliche Mehrfachzuordnung innerhalb einer Facette gibt. Des Weiteren wurden acht Methoden und deren Zuordnung als Beispiel angegeben, um die praktische Umsetzung der Klassifikation zu demonstrieren (Siehe Tabelle 1 und 2). 


\section{Diskussion und Ausblick}

Es konnten Merkmale zur Klassifikation extrahiert und auf deren Grundlage ein Schema erstellt werden, dass der Ein- und Zuordnung von Usability-Evaluationsmethoden dient. Im Kontrast zu den bestehenden Klassifikationsansätzen bietet dieses Klassifikationsschema mehr Möglichkeiten zur genaueren Gegenüberstellung der Evaluationsverfahren. Entscheidend ist in diesen Zusammenhang die Klassifikationsstruktur, die in Form einer postkombinierten Klassifikation (Facettenklassifikation) vorliegt. Dadurch können die Elemente einer Struktur gleichzeitig aus verschiedenen Perspektiven betrachtet werden. Dennoch werden dabei verhältnismäßig weniger Klassen benötigt als bei einer Hierarchie, in der die gleiche Menge an Informationen strukturiert ist (Arndt 2006: 159 ff.). Die Anwender einer Facettenklassifikation werden somit niemals mit einer unübersichtlichen mehrdimensionalen Struktur konfrontiert. Die Erweiterung und Pflege von Facettenklassifikation gestaltet sich ebenfalls einfacher als Klassifikationen, die auf einer anderen Grundstruktur aufgebaut sind. Für die neu entstandene Klassifikation konnten insgesamt zehn Klassifikationsmerkmale gefunden und anhand von existierenden Methoden erfolgreich erprobt werden. Damit bietet sie einen höheren Informationsgehalt als bisherige Klassifikationen zu Usability-Evaluationsmethoden. Es bleibt zu prüfen, ob sich die einzelnen Klassen innerhalb der Facetten zur Einordnung von Usability-Evaluationsmethoden eignen. Bisher ist das Schema noch nicht evaluiert und zur Bewertung sollen Usability-Experten wie auch Nicht-Experten, dass oben aufgeführte Ordnungssystem an einer Vielzahl von Methoden anwenden. Die Ergebnisse können dann in einer Klassifikationstabelle bzw. Wahrheitsmatrix zusammengefasst werden. Anschließend wäre es, durch die Berechnung der relativen Häufigkeiten aus den vorliegenden Werten möglich, Kenngrößen zur Beurteilung der Klasse zu ermitteln. Ergänzend muss angemerkt werden, dass sich Facettenklassifikationen sehr gut als Grundlage für den Aufbau von strukturierten Wissenssammlungen eignen, aber keine Beziehungen und Hierarchien abbilden. Zur logischen Erschließung von Zusammenhängen sind für Neueinsteiger in das Themenfeld andere Klassifikationsansätze womöglich besser geeignet. Als Ausgangspunkt für die Entwicklung einer standardisierten Auswahlsystematik für UsabilityEvaluationsmethoden stellt die neue Klassifikation hingegen einen wichtigen Zwischenschritt dar. Ergänzend zur logischen Einteilung der Methoden werden nun im nächsten Schritt Beurteilungskriterien definiert, wie beispielsweise zeitlicher und materieller Aufwand der Evaluation oder notwendige Qualifikation der Evaluatoren (Sarodnick \& Brau 2011: S.199 ff.). Genau an dieser Entwicklung einer standardisierten Auswahlsystematik arbeiten die Autoren, um bei der Usability-Bewertung in Software-Projekten ein gut nutzbares Instrument zur Auswahl der „richtigen“ Methode anzubieten.

\section{Literaturverzeichnis}

Arndt, H. (2006). Integrierte Informationsarchitektur: Die erfolgreiche Konzeption professioneller Websites. 1. Auflage, Berlin: Springer.

Bortz, J., \& Döring, N. (2006). Forschungsmethoden und Evaluation: für Human- und Sozialwissenschaftler. 4. Auflage. Berlin: Springer. 
Christos, F., Christos, K., Eleftherios, P., Nikolaos, T., \& Nikolaos, A. (2007). Remote Usability Evaluation Methods and Tools: A Survey. Proceedings of the 11th Panhellenic Conference in Informatics (PCI 2007), $151-162$.

DIN EN ISO 9241-11 (1998). Ergonomische Anforderungen für Bürotätigkeiten mit Bildschirmgeräten - Teil 11: Anforderungen an die Gebrauchstauglichkeit. Beuth, Berlin.

DIN EN ISO 9241-210 (2010). Ergonomie der Mensch-System-Interaktion - Teil 210: Prozess zur Gestaltung gebrauchstauglicher interaktiver Systeme. Beuth, Berlin.

Freymann, M. (2008). Ein Tool zur Klassifikation und Auswahl von Evaluations-methoden im UserCentred Design Prozess. In K. Röse \& H. Brau (Hrsg.): Mensch und Computer 2008. Usability Professionals 2007.

Ivory, M. Y., \& Hearst, M. (2001). The state of the art in automating usability evaluation of user interfaces. ACM Computing Surveys, 33(4), 470-516.

Krannich, D. (2010). Mobile System Design: Herausforderungen, Anforderungen und Lösungsansätze für Design, Implementierung und Usability-Testing Mobiler Systeme. 1.Auflage. Norderstedt: Books on Demand.

Law, E. L.-chong, Scapin, D., Cockton, G., Springett, M., Stary, C., \& Winckler, M. (Hrsg.) (2009). Maturation of Usability Evaluation Methods : Retrospect and Prospect. Final Reports of COST294MAUSE Working Groups. IRIT Press, Toulouse.

Ohl, C. (2014). Klassifikationsschemen für UEMs. URL: http://de.slideshare.net/ChristophOhl/klassifikationsschemen-fur-uems. (Stand 18.06.2015)

Sarodnick, F., \& Brau, H. (2011). Methoden der Usability Evaluation. E. Bamberg, G. Mohr, \& M. Rummel (Hrsg.). 2.Auflage. Hans Huber, Bern.

Seffah, A., \& Metzker, E. (2009). Adoption-centric Usability Engineering: Systematic Deployment, Assessment and Improvement of Usability Methods in Software Engineering. Springer, London.

Whitefield, A., Wilson, F., \& Dowell, J. (1991). A framework for human factors evaluation. Behaviour \& Information Technology, 10(1), 65-79.

Woywode, M., Mädche, A.,Wallach, D. \& Plach, M. (2012). Abschlussbericht des Forschungsprojekts: Gebrauchstauglichkeit von Anwendungssoftware als Wettbewerbsfaktor für kleine und mittlere Unternehmen (KMU). ifm - Institut für Mittelstandsforschung Universität Mannheim.

Zhang, Z. (2007). Usability Evaluation. In P. Zaphiris \& S. Kurniawan (Hrsg.): Human Computer Interaction Research in Web Design and Evaluation. Idea Group Publishing, London, S.209-288.

Zimmermann, D., \& Grötzbach, L. (2007). A requirement engineering approach to user centered design. Proceedings of the 12th international Conference on Human-computer interaction: Interaction Design and Usability, 4550(4), 360 - 369.

\section{Kontaktinformationen}

Christoph Ohl (christoph.ohl@gmail.com)

Gabriele Schade (schade@fh-erfurt.de) 